\title{
Deteç̧ão de Malwares Android: Levantamento Empírico da Disponibilidade e da Atualização das Fontes de Dados
}

\author{
Tainá Soares $^{1}$, Joner Mello ${ }^{1}$, Lucas Barcellos ${ }^{1}$, Renato Sayyed ${ }^{1}$, Guilherme Siqueira ${ }^{1}$ \\ Karina Casola $^{1}$, Estevão Costa ${ }^{2}$, Nicolas Gustavo ${ }^{2}$, Eduardo Feitosa ${ }^{2}$, Diego Kreutz ${ }^{1}$, \\ ${ }^{1}$ Universidade Federal do Pampa (Unipampa) \\ ${ }^{2}$ Universidade Federal do Amazonas (UFAM)
}

\begin{abstract}
Resumo. Neste estudo avaliamos 84 fontes de dados utilizadas para a concepção de modelos de aprendizado de máquina aplicados à detecção de malwares Android, sendo 39 lojas de aplicativos, 30 datasets e 15 repositórios de APKs. Verificamos que 68,75\% dos trabalhos utilizam fontes de dados antigas, mesmo existindo opções de fontes atuais. Também observamos que a disponibilidade e a corretude dos registros das fontes de dados nem sempre são condizentes com o informado e, consequentemente, podem impactar negativamente a qualidade dos métodos de detecção de malwares.
\end{abstract}

\section{Introdução}

Para modelos de detecção de malwares Android, a atualidade dos datasets é importante e pode impactar diretamente o desempenho da solução [Allix et al., 2015]. Como os malwares Android possuem uma natureza dinâmica, modelos de aprendizado de máquina conseguem reconhecer um aplicativo malicioso atual somente se os dados de treino incluírem informações sobre o comportamento de malwares atuais.

Na prática, muitos datasets atuais são construídos a partir de datasets mais antigos [Sharma and Rattan, 2021], o que também representa um problema. Por exemplo, o dataset Drebin-215 (disponibilizado em 2018) é constituído, na verdade, por um subconjunto de dados do dataset Drebin, datado de 2012. O mesmo ocorre com diversos outros conjuntos de dados, como o Android Botnet, formado por dados do Malware Genome Project, Contagio Mini Dump e VirusTotal. Além disso, há datasets, como o CICInvesAndMal2019, que afirmam incluir dados atuais, de 2019, porém, contêm apenas características de versões bastante antigas (e.g., 2016 e inferior) da API do Android.

Neste trabalho temos como objetivos: (a) realizar um levantamento detalhado de informações sobre a atualização e a disponibilidade de fontes de dados; e (b) investigar as fontes de dados utilizadas na prática por trabalhos que propõem modelos de aprendizado de máquina para detecção de malwares Android. Como contribuições resultantes do desenvolvimento destes objetivos, podemos destacar: (a) catalogação e classificação de 84 fontes de dados com relação ao tipo, disponibilidade e atualização; (b) avaliação e discussão sobre as fontes de dados utilizadas nas pesquisas de 35 trabalhos acadêmicos; (c) identificação de inconsistências na informação de atualização das fontes de dados; e (d) identificação de obstáculos à reprodutibilidade dos trabalhos.

Em estudos similares, como o [Kouliaridis et al., 2020], os autores limitam-se ao estudo de 10 datasets, onde comparam características como idade, tamanho, acesso (disponível, indisponível ou através de solicitação) e destacam a presença de características como permissões e intents em $30 \%$ dos datasets. O nosso estudo cobre um número mais 
expressivo de fontes de dados (84) e investiga detalhadamente questões relacionadas à disponibilidade e atualização das fontes utilizadas em estudos atuais.

O trabalho está organizado como segue. Nas Seções 2 e 3 , apresentamos as 84 fontes de dados catalogadas e uma discussão sobre as fontes de dados utilizadas nos 35 trabalhos analisados, respectivamente. Finalmente, nas Seções 4 e 5 , apresentamos questões sobre a atualização das fontes de dados e as considerações finais, respectivamente.

\section{Atualização e Disponibilidade das Fontes de Dados}

O conjunto de dados analisado neste trabalho é composto por 84 fontes, sendo 39 lojas de aplicativos Android, 30 datasets e 15 repositórios de Pacotes de Aplicação Android (Android Application Pack ou simplesmente APKs). Essas fontes foram catalogadas a partir: (a) da revisão sistemática sobre detecção de aplicações maliciosas no Android [Sharma and Rattan, 2021], (b) de 35 trabalhos selecionados para análise (conforme detalhado em [Soares et al., 2021b]) e (c) dos 100 primeiros resultados da busca por "Android Dataset" no Google Dataset Search ${ }^{1}$, Kaggle ${ }^{2}$ e FigShare ${ }^{3}$. A relação completa e detalhada das fontes está disponível na versão estendida do trabalho [Soares et al., 2021a].

As fontes foram classificadas, em relação à disponibilidade, em três tipos: disponível, indisponível e restrito. Para realizar essa classificação, foram utilizadas as duas convenções a seguir: Fontes não localizadas são aquelas não encontradas nos nossos processos de busca, que foram (a) buscas web pelo nome da fonte e (b) verificação de todos os links retornados das duas primeiras páginas de resultado. As buscas, por cada fonte de dados, foram realizadas por, no mínimo, dois co-autores do trabalho. Fontes sem acesso público são aquelas sendo encontradas através das nossas buscas, isto é, o link da fonte foi encontrado, mas na página (a) não há informações sobre como acessar os dados (e.g., se é necessário enviar um e-mail, preencher um formulário ou solicitar previamente algum tipo de autorização) ou (b) é informado que a fonte não está mais disponível.

A classificação de uma fonte como disponível significa que ela foi localizada e seu acesso é público, isto é, sem restrições (e.g., autorização prévia ou credenciais de acesso). As fontes não localizadas ou sem acesso público foram classificadas como indisponíveis, como é o caso do Malware Genome Dataset. Finalmente, todas as fontes que exigem alguma autorização prévia (e.g., contato via e-mail ou formulário) ou credenciais (e.g., login e senha) para o acesso foram classificadas como restritas. A classificação quanto a disponibilidade das fontes foi baseada no trabalho de [Kouliaridis et al., 2020], que classifica 10 datasets.

As fontes que requerem solicitação de acesso, mas não responderam às solicitações em 30 dias ou mais, como é o caso do The Drebin Dataset, e aquelas que exigem credenciais mediante pagamento, como é o caso da Virus Total Malware Service Intelligence, foram classificadas como indisponíveis.

\subsection{Lojas de Aplicativos}

As lojas (ou mercados) de aplicativos são plataformas que servem como meio de distribuição de software para dispositivos móveis, como smartphones e tablets, baseados

1 https://datasetsearch.research.google.com/

2 https://www.kaggle.com/

3 https://figshare.com/ 
em Android. Essas lojas armazenam o APK de cada aplicativo disponibilizado por elas, que é utilizado para instalar o aplicativo nos dispositivos. O principal exemplo de mercado de aplicativos Android é a Google Play Store, loja oficial para o sistema operacional Android.

Das 39 lojas de aplicativos, 25 foram classificadas como disponíveis e destas, $84 \%$ (21 delas) são atualizadas constantemente. As lojas disponíveis são aquelas que possuem um site oficial acessível e alguma forma (e.g., link) para o download dos APKs, como é o caso do mercado AndroidLista. Por outro lado, há 14 lojas que classificamos como indisponíveis por não terem um meio de acesso aos aplicativos. Na maior parte dos casos, como o AndroidDrawer, há problemas no acesso do site da loja - site não abre ou retorna erro. Há casos de lojas, como a GFan, Anruan e 10086, onde os sites oficiais retornam erro de servidor não encontrado para diferentes navegadores web populares (e.g., Google Chrome, Mozilla Firefox) e, foram classificadas como indisponíveis.

\subsection{Datasets e Repositórios de APKs}

A classificação quanto a atualização dos datasets e repositórios de APKs foi realizada utilizando intervalos de tempo ([2008-2012], [2013-2017] e [2018-2021]) para agrupar os dados. As datas consideradas são aquelas informadas nos estudos que originaram as fontes ou nos sites delas. Se a data informada é 2015, como no caso do Wang's Repository, classificamos a atualização da fonte como contida no intervalo [2013-2017]. Os detalhes completos, de todos os repositórios e datasets, podem ser vistos em [Soares et al., 2021a].

Dos 45 datasets e repositórios, 25 são disponíveis, 11 são restritos e 9 são indisponíveis. Do total, 20 podem ser considerados como atualizados, isto é, estão contidos no intervalo de 2018 e 2021 (parâmetro utilizado neste estudo). Outra observação interessante é o fato de a maioria das fontes consideradas disponíveis serem também as mais atuais: 16 de um total de 25 têm atualização entre 2018 e 2021, conforme pode ser observado na Tabela 1 .

Um resumo do período de atualização dos datasets e dos repositórios de APKs classificados como disponíveis ou restritos pode ser visto no gráfico da Figura 1. Cerca de 55\% dessas fontes (conjunto das disponíveis ou restritas) têm atualização entre 2018 e 2021, ou seja, mais da metade são consideradas recentes. As informações de disponibilidade e quantidade das fontes de dados dispostas por período de atualização sugerem haver uma tendência em tornar os dados públicos e de fácil acesso.

Há alguns casos de fontes de dados disponíveis (e.g., CICMalDroid 2020) que disponibilizam tanto datasets quanto repositórios de APKs. Outra característica dessa fonte é que ela é composta por amostras de malwares coletadas de diversas outras fontes: VirusTotal, AMD, contagio, entre outras. Além do CICMalDroid 2020, outras fontes também contêm dados de diversas origens, como é o caso do Android Botnet e CICAndMal2017.

\section{Trabalhos e Fontes de Dados}

Anteriormente, analisamos a reprodutibilidade de 35 artigos científicos [Soares et al., 2021b]. Neste trabalho, realizamos uma análise das 31 fontes de dados utilizadas pelos trabalhos, das quais 11 são lojas de aplicativos e 20 são datasets ou repositórios de APKs. 
Tabela 1. Datasets e Repositórios de APKs: Atualização e Disponibilidade

\begin{tabular}{l|c}
\hline \hline Datasets e Repositórios de APKs & Atualização \\
\hline Ether Malware Analysis Dataset & {$[2008-2012]$} \\
\hline Contagio Malware Dump, CIC-AAGM2017, MudFlow, Android Botnet, & Acesso \\
M0Droid, GaziBenignApp, Heldroid & \\
\hline Contagio Mobile, Android Permissions Dataset, VirusShare, & \\
CICInvesAndMal2019, TheZoo, AndroMalShare, Koodous, Drebin-215, & [2018-2021] \\
Dataset of Android Permissions, CICMalDroid 2020, & \\
Android Malware and Normal Permissions Dataset, & \\
Android Malware and Benign Application Dataset,, Disponível \\
$\begin{array}{l}\text { PARUDroid, Comodo Cloud Security Center, Wang's Repository, } \\
\text { Drebin4000 and AMD6000 }\end{array}$ \\
\hline MobileSandbox project (MobWorm) & Atualização Não Encontrada \\
\hline CIC-AndMal2017, AndroZoo, Andro-AutoPsy, Andro-Dumpsys, & {$[2013-2017]$} \\
Andro-Profiler, Andro-Tracker & Atualização Não Encontrada \\
\hline UpDroid, Contagio Mini Dump, COVID19 Apps, CCCS-CIC-AndMal-2020 & {$[2008-2012]$} \\
\hline DroidKin & {$[2013-2017]$} \\
\hline The Drebin Dataset, Android Malware Genome Project & Restrito \\
\hline Android PRAGuard Dataset, PlayDrone Project & Atualização Não Encontrada \\
\hline $\begin{array}{l}\text { McAfee, Inter-Component Communication (IcRE) Repository, } \\
\text { New Malware Families 2015, Virus Total Malware Service Intelligence, }\end{array}$ & Indisponível \\
Kharon Malware Dataset & \\
\hline \hline
\end{tabular}

Os repositórios de APKs estão em itálico. Fontes que disponibilizam tanto datasets quanto repositórios de APKs estão em negrito.

\subsection{Lojas de Aplicativos}

As lojas de aplicativos são comumente utilizadas como fonte de aplicativos benignos para compor os datasets [Wang et al., 2019]. Em 21 dos 35 trabalhos analisados, os datasets têm dados oriundos de lojas, como Google Play Store, SlideME e PandaApp. Do total de 11 lojas, 8 foram classificadas como disponíveis e todas, exceto a SlideME (http://slideme.org/), são atualizadas constantemente. A disponibilidade detalhada dessas e das demais lojas pode ser vista em [Soares et al., 2021a]. A SlideME incluiu um aplicativo novo pela última vez em 2019, mas em 2021 atualizou um aplicativo da sua loja.

A disponibilidade e atualização da maioria das lojas pode sugerir que os dados utilizados pelos trabalhos são atuais e de fácil acesso. Infelizmente, todos os trabalhos falham em relação ao acesso, pois não fornecem os dados necessários sobre os aplicativos, como os nomes e versões, que são fundamentais para a reprodutibilidade dos trabalhos.

\subsection{Datasets e Repositórios de APKs}

Dos 20 datasets e repositórios de APKs, 45\% foram classificados como disponíveis, $40 \%$ como indisponíveis e $15 \%$ como acesso restrito. É interessante observar que $65 \%$ dos trabalhos utilizam pelo menos uma das 8 fontes indisponíveis.

Considerando os 16 trabalhos mais recentes, publicados de 2018 a 2021, podemos verificar a atualização e a disponibilidade das fontes de dados na Figura 1. Como pode ser observado, 12 dos 16 trabalhos citam alguma fonte de dados atual (em relação aos seus respectivos anos de publicação) disponível ou restrita. Entretanto, todos os trabalhos utilizam pelo menos uma fonte de dados com atualização entre 2012 e 2016. Consequentemente, podemos concluir que nenhum desses trabalhos utiliza um conjunto de dados atualizado, pois todos os datasets contêm também dados de fontes defasadas, i.e., antigas. 

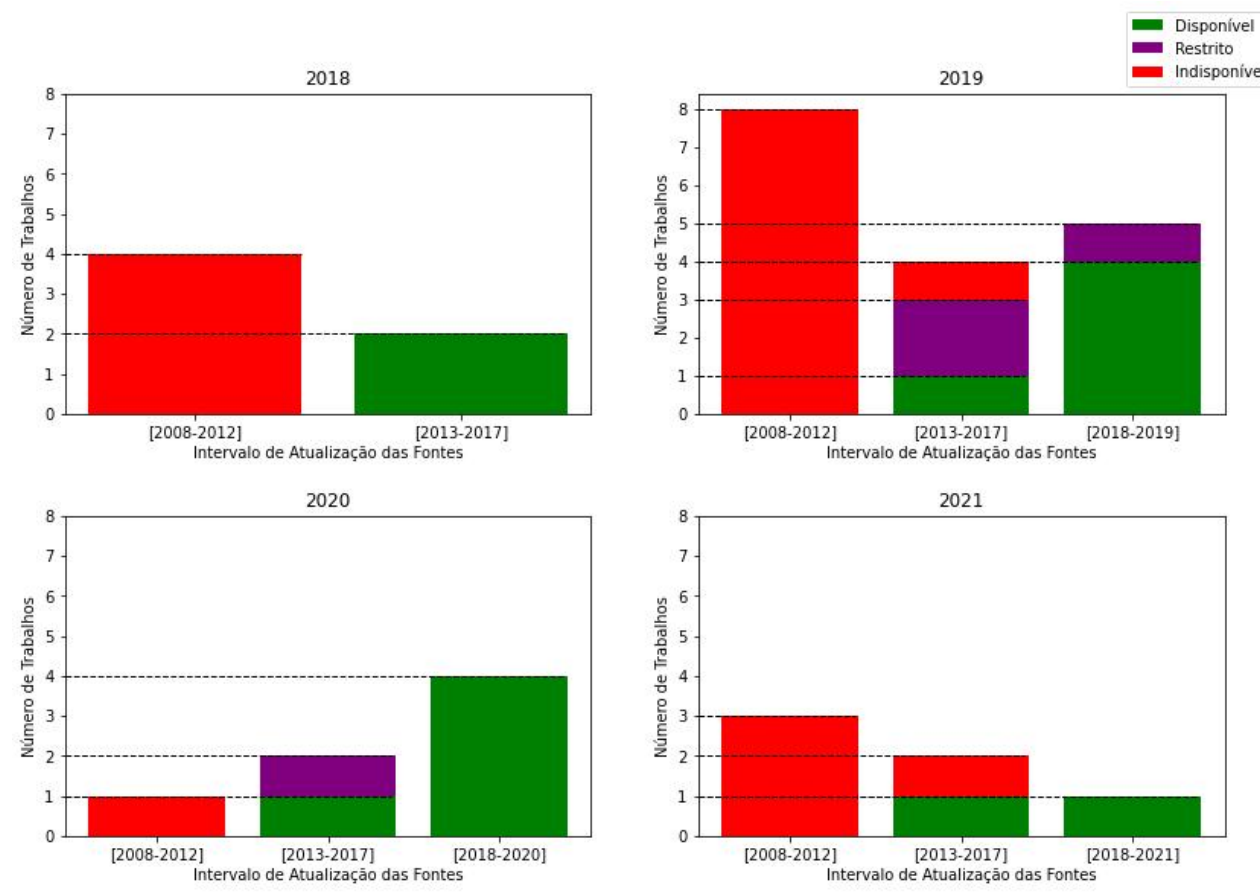

Figura 1. Atualização e Disponibilidade das Fontes de Dados Utilizadas

\section{Atualização das Fontes de Dados}

Um ponto a ser analisado acerca das datas das fontes é a possibilidade de diferença entre as datas informadas nos trabalhos ou sites e as datas das APIs presentes em determinada fonte de dados. Esse é o caso das fontes CIC-InvesAndMal2019 4 e CICMalDroid2020 5 .

As fontes de dados CIC-InvesAndMal2019 e CICMalDroid2020 foram analisadas quanto às versões das APIs da seguinte maneira: (i) primeiramente, o download dos APKs de cada uma delas foi realizado; (ii) depois disso, um algoritmo que seleciona os APKs válidos foi executado sobre os dados, pois há alguns arquivos APK corrompidos (i.e. mal formados, impossíveis de serem analisados); (iii) por fim, foram verificadas as APIs presentes nos arquivos válidos.

O CIC-InvesAndMal2019 teve os dados coletados de 2012 a 2019 e, portanto, tem 2019 como ano de atualização. A questão é que essa fonte contém APIs de versões de antes de 2012 e não contém nenhuma API de 2019. Ou seja, o CIC-InvesAndMal2019 é considerada uma fonte de 2019, mas não contém dados de APIs de 2019 (a mais recente é de 2016). Já no caso do CICMalDroid2020, foi verificada a presença de dados de aplicações de APIs atuais, mas a grande maioria destas são aplicações benignas. Este também é um problema, pois por mais que seja verdade que a fonte contenha dados de APIs atuais, ela continua sendo desatualizada quanto às aplicações malignas, que são a parte mais importante quando o contexto é o uso desses dados para detecção de malwares e aprendizado de máquina.

\footnotetext{
${ }^{4}$ https://www.unb.ca/cic/datasets/invesandmal2019.html 5 https://www.unb.ca/cic/datasets/maldroid-2020.html
} 


\section{Considerações Finais}

As principais conclusões do nosso estudo podem ser separadas de acordo com as duas análises realizadas: (a) panorama das 84 fontes de dados e (b) uso das fontes de dados por parte dos 35 trabalhos. Com relação à análise (a), onde foram considerados aspectos de atualização e disponibilidade de 84 fontes de dados, as principais conclusões são: (i) a maioria $(59,52 \%)$ das fontes de dados são disponíveis (aproximadamente $55 \%$ dos datasets e repositórios de APKs e 64\% dos mercados de aplicativos Android); e (ii) existe uma tendência em tornar as fontes de dados disponíveis, pois quanto mais atual o período de tempo analisado, maior a quantidade de fontes disponíveis.

Já quanto a análise (b), podemos concluir que as fontes de dados desatualizadas e indisponíveis ainda são bastante utilizadas em pesquisas atuais da área. Esse fato é um problema, pois um modelo de aprendizado de máquina não pode garantir a identificação de uma nova linhagem de malware que não é representada no conjunto de dados de treinamento [Allix et al., 2015]. A atualização regular de datasets e a proximidade histórica destes conjuntos de dados são fundamentais para a minimizar as ameaças à validade destes estudos.

Como trabalhos futuros podemos destacar: (a) analisar as versões das APIs presentes nas fontes de dados para validar a atualização; (b) realizar o mapeamento de relação de origem entre as fontes de dados para verificar o quão novas as fontes são; (c) avaliar o impacto de datasets de diferentes períodos nos modelos de detecção de malwares; e (d) analisar as fontes de dados de acordo com a possibilidade de acesso às versões antigas das aplicações.

\section{Agradecimentos}

Esta pesquisa foi financiada, conforme previsto nos Arts. 21 e 22 do decreto no. 10.521/2020, nos termos da Lei Federal no. 8.387/1991, através do convênio no. 003/2021, firmado entre ICOMP/UFAM, Flextronics da Amazônia Ltda e Motorola Mobility Comércio de Produtos Eletrônicos Ltda.

\section{Referências}

Allix, K., Bissyandé, T. F., Klein, J., and Le Traon, Y. (2015). Are your training datasets yet relevant? In Piessens, F., Caballero, J., and Bielova, N., editors, Engineering Secure Software and Systems, pages 51-67, Cham. Springer International Publishing.

Kouliaridis, V., Kambourakis, G., and Peng, T. (2020). Feature importance in mobile malware detection. CoRR, abs/2008.05299.

Sharma, T. and Rattan, D. (2021). Malicious application detection in android - a systematic literature review. Computer Science Review, 40:100373.

Soares, T., Mello, J., Barcellos, L., Sayyed, R., Siqueira, G., Casola, K., Costa, E., Gustavo, N., Feitosa, E., and Kreutz, D. (2021a). Detecção de malwares android: Levantamento empírico da disponibilidade e da atualização das fontes de dados (versão estendida). https: / / arxiv.kreutz.xyz/wrseg2021_ disponibilidade_vel.pdf.

Soares, T., Siqueira, G., Barcellos, L., Sayyed, R., Vargas, L., Rodrigues, G., Assolin, J., Pontes, J., and Kreutz, D. (2021b). Detecção de malwares android: datasets e reprodutibilidade. https://arxiv . kreutz.xyz/mh21_reprodutibilidade.pdf.

Wang, S., Chen, Z., Yan, Q., Yang, B., Peng, L., and Jia, Z. (2019). A mobile malware detection method using behavior features in network traffic. Journal of Network and Computer Applications, 133:15-25. 